\title{
Lattice Reactions Governing Thermoelasticity and Superelasticity in Shape Memory Alloys
}

\section{Adiguzel 0*}

Department of Physics, Firat University, Turkey

*Corresponding author: Osman Adiguzel, Department of Physics, Firat University, Turkey, Tel: +905365638403; Email: oadiguzel@firat.edu.tr

\section{Mini Review \\ Volume 5 Issue 1}

Received Date: April 08, 2021

Published Date: April 19, 2021

DOI: $10.23880 / p s b j-16000170$

\section{Abstract}

Some materials take place in class of advanced smart materials with adaptive properties and stimulus response to the external changes. Shape memory alloys take place in this group, due to the shape reversibility and capacity of responding to changes in the environment. These alloys exhibit a peculiar property called shape memory effect, which is characterized by the recoverability of two certain shapes of material at different temperatures. These alloys have dual characteristics called thermoelasticity and superelasticity, from viewpoint of memory behavior, which is governed by two structural transformations, thermal and stress induced martensitic transformations performed by lattice twinning and detwinning reactions. Thermal induced transformation is governed by lattice twinning and occurs on cooling and ordered parent phase structures turn into twinned martensite structures, and these twinned structures turn into detwinned martensite structures by means of strain induced martensitic transformation, by stressing material in low temperature condition. Superelasticity is performed by stressing and releasing material at a constant temperature in parent phase region, and shape recovery is performed simultaneously upon releasing the applied stress.

Keywords: Thermoelasticity; Superelasticity; Alloys; Martensite

\section{Introduction}

Shape memory alloys are smart and functional materials due to the response to the external condition like temperature change and mechanically deformation. These alloys are used as shape memory elements in many fields of industry, engineering, aircraft industry, biomedical and other sectors [1,2]. Shape memory effect is initiated by cooling and deformation processes in shape memory alloys and performed thermally on heating and cooling after these processes. Therefore, this behavior can be called thermoelasticity. These alloys are plastically deformed at the low temperature condition, strain energy is stored in the material by keeping the deformed shape after releasing the external forces, and releases upon heating by recovering the original shape. Also, the shape of the material cycles in reversible way between the deformed and original shapes on cooling and heating.

Thermoelasticity and superelasticity is based on crystallographic transformations called thermal and stress induced martensitic transformations. Shape memory effect is result of successive thermal and stress induced martensitic transformations, superelasticity is governed only mechanically by the strain induced transformation by stressing and releasing material at a constant temperature in parent beta phase region.

Thermal induced martensite occurs along with lattice twinning on cooling and ordered parent phase structures turn into twinned martensite structures, and these twinned structures turn into detwinned martensite structures 
by means of strain induced martensitic transformation, by stressing material in low temperature condition. Superelasticity is performed by stressing and releasing material at a constant temperature in parent phase region, and shape recovery is performed simultaneously upon releasing the applied stress. Superelasticity exhibits classical elastic material behavior by recovering the original shape after releasing. Stressing and releasing paths are different at the stress-strain diagram and the cycling loop refers to the energy dissipation. Superelasticity is also result of the stress induced martensitic transformation and ordered parent phase structures turn into the detwinned martensite structures.

Thermal induced transformations occur as martensite variants with cooperative movements of atoms by means of lattice invariant shears on a $\{110\}$-type plane of austenite matrix, and the transformed region consists of parallel bands containing alternately two different variants. These variants form internally twinned martensite regions. In the absence of external stress, multiple martensite variants are formed without causing any macroscopic deformation as lattice twins in self-accommodating manner [3].

\section{Experimental}

Experimental studies have been performed on two copper based ternary shape memory alloys, with the following compositions: $\mathrm{Cu}-26.1 \% \mathrm{Zn}-4 \% \mathrm{Al}$ and $\mathrm{Cu}-11 \% \mathrm{Al}-$ $6 \% \mathrm{Mn}$ (in weight). Martensitic transformation temperatures of these alloys are over the room temperature and they are entirely martensitic state at room temperature. Specimens of these alloys were solution treated for homogenization in the $\beta$-phase field $\left(15\right.$ minutes at $830^{\circ} \mathrm{C}$ for CuZnAl alloy and 20 minutes at $700^{\circ} \mathrm{C}$ for CuAlMn alloy), then quenched in icedbrine to retain the $\beta$-phase and aged at room temperature after quenching. Powder specimens for x-ray examination were prepared by filling the alloys, these specimens were heated in evacuated quartz tubes in the $\beta$-phase fields for homogenization and quenched in iced-brine. X-ray diffraction studies carried out on these specimens, and x-ray diffraction profiles were taken from the quenched specimens using $\mathrm{Cu}$ $\mathrm{K}_{\alpha}$ radiation with wavelength 1.5418 Å. Powder samples of the alloys were aged at room temperature, and a series of x-ray diffractograms were taken in a long-term interval and compared with each other.

\section{Results and Discussion}

X-ray powder diffractograms and electron diffractions were taken from the samples in a long-term interval. Two $\mathrm{x}$-ray powder diffractogram taken from the long term aged CuZnAl and CuAlMn alloy samples are shown in Figure 1 and Figure 2. These diffractograms have been indexed on the monoclinic M18R basis. Two electron diffraction patterns taken from the samples of both alloys are shown in Figure 3. $\mathrm{X}$-ray diffractograms and electron diffraction patterns reveal that both alloys have the ordered structure in martensitic condition, and exhibit superlattice reflections, the details were given elsewhere [3]. As seen at the x-ray diffractograms, peak intensities are different exhibit super lattice reflection. Parent phases of Copper-based ternary alloys have $\mathrm{DO}_{3}$-type ordered structure, and this structure turns into 18R-type layered structures with thermal induced transformation on cooling [3].

A series of X-ray powder diffractograms and electron diffraction patterns were taken from both CuZnAl and CuAlMn alloy samples in a large time interval and compared with each other. It has been observed that electron diffraction patterns exhibit similar characteristics, but some changes occur at the diffraction angles and intensities on the x-ray diffractograms with aging duration. These changes occur as rearrangement or redistribution of atoms in the material, and attribute to new transitions in diffusive manner [3-5].

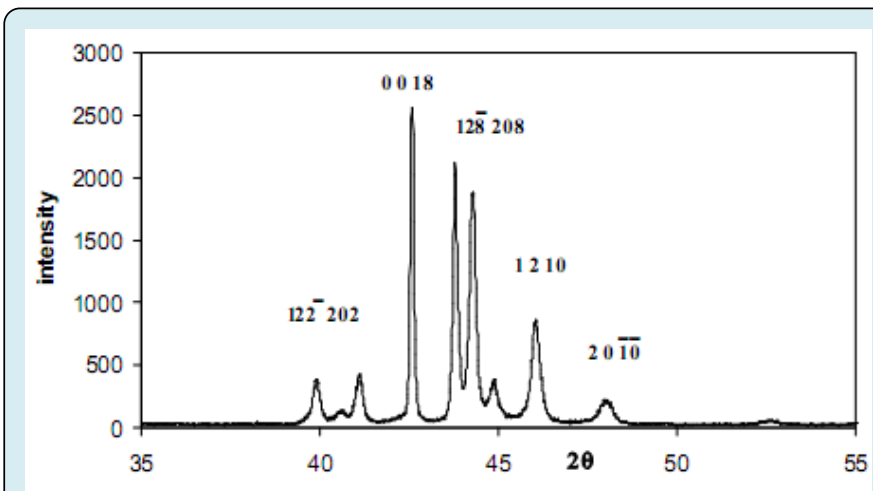

Figure 1: An x-ray diffractogram taken from the long term aged CuZnAl alloy sample.

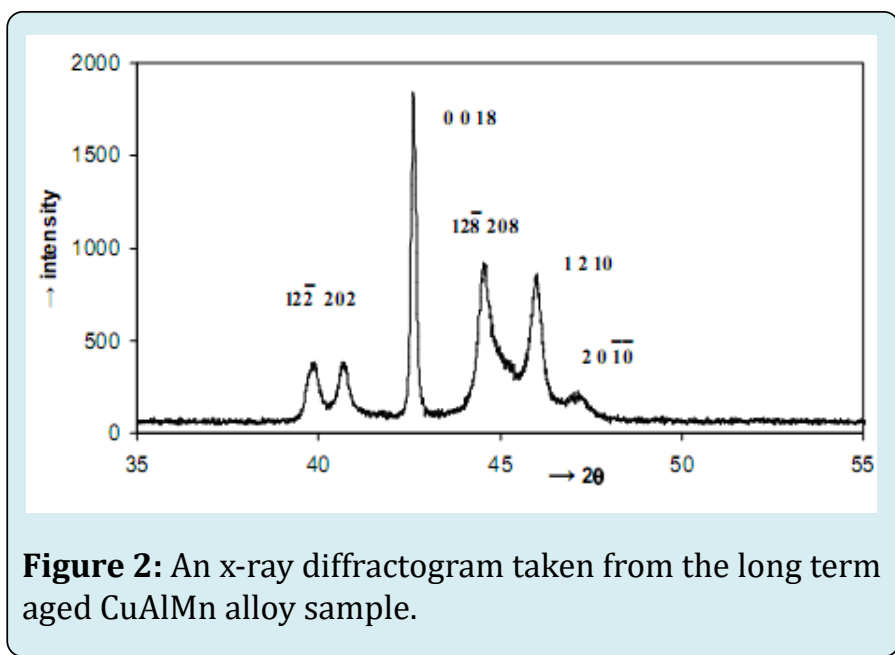



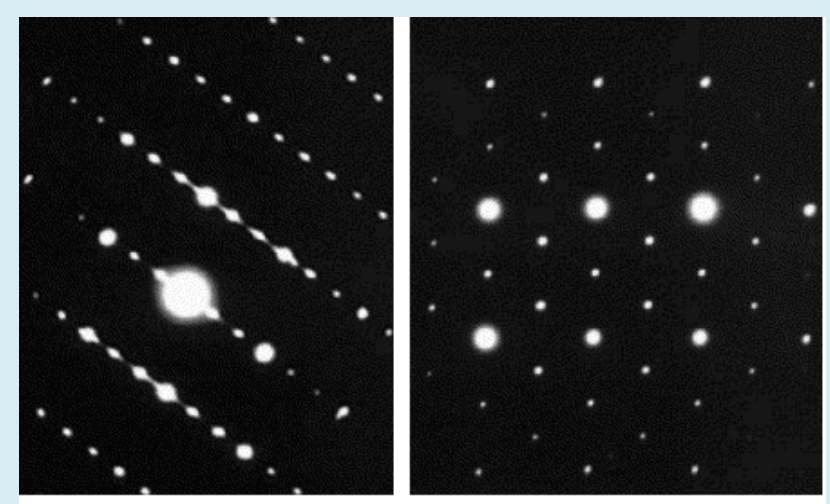

Figure 3: Two Electron diffraction patterns taken from the $\mathrm{CuZnAl}$ and CuAlMn alloy samples.

In particular, some of the neighbor peak pairs have moved toward each other. It is interesting that miller indices of these plane pairs provide a special relation: $\left(h_{1}^{2}-h_{2}^{2}\right) / 3=\left(k_{2}^{2}-k_{1}^{2}\right) / n$, where $\mathrm{n}=4$ for $18 \mathrm{R}$ martensite [6].

These plane pairs can be listed as follow; (122)-(202), (128)(208), (1 2 10) - (2 0 10), (040)- (320). This result can be attributed to a relation between interplane distances of these plane pairs and rearrangement of atoms on the basal plane. In these changes, atom sizes play important role.

The ordered structure or super lattice structure is essential for the shape memory quality of the material. In the shape memory alloys, homogenization and releasing the external effect is obtained by ageing at $\beta$ - phase field for adequate duration.

It can be concluded from the above results that the copper-based shape memory alloys are very sensitive to the ageing treatments. The diffraction angles and intensities of x-ray diffraction peaks changes with the ageing time in martensitic condition. Some of the successive peak pairs providing a special relation between Miller indices come close each other. These changes referee to the martensite stabilization in the redistribution or disordering manner, and stabilization proceeds by a diffusion-controlled process. The martensite stabilization is a diffusion-controlled phenomenon and leads to redistribution of atoms on the lattices sites, although martensitic transformation has displacive character. The ordered structure or super lattice structure is essential for the shape memory quality of the material.

\section{References}

1. Barbarino S, Saavedra Flores EI, Ajaj RM, Dayyani I, Friswell MI (2014) A Review on Shape Memory Alloys with Applications to Morphing Aircraft. Smart Mater Struct 23(6): 1-190.

2. Petrini L, Migliavacca F (2011) Biomedical Applications of Shape Memory Alloys. Journal of Metallurgy 2011: 501483.

3. Adiguzel 0 (2013) Phase Transitions and Microstructural Processes in Shape Memory Alloys. Materials Science Forum 762: 483-486.

4. Li Z, Gang S, Wang MP (2008) Macroscopic shape change of Cu13Zn15Al shape memory alloy on successive heating, Journal of Alloys and Compounds 452(2): 307311.

5. De Castro F, Sade M, Lovey F (2012) Improvements in the Mechanical Properties of the 18R $\leftrightarrow$ R High-hysteresis Martensitic Transformation by Nanoprecipitates in CuZnAl Alloys. Materials Science and Engineering A 543: 88-95.

6. Adiguzel 0 (2015) The Role of Twinned and Detwinned Structures on Memory Behavior of Shape Memory Alloys. Advanced Materials Research 1105: 78-82. 